\title{
BMJ Open Preventing adverse cardiac events (PACE) in chronic obstructive pulmonary disease (COPD): study protocol for a double-blind, placebo controlled, randomised controlled trial of bisoprolol in COPD
}

\author{
Allison Martin (D) , ${ }^{1,2}$ Robert J Hancox,${ }^{3}$ Catherina L Chang, ${ }^{4}$ Richard Beasley (D) ${ }^{5}$ \\ Jeremy Wrobel, ${ }^{6,7}$ Vanessa McDonald, ${ }^{8,9}$ Claudia C Dobler (D) , ${ }^{1,2,10}$ lan A Yang, ${ }^{11,12}$ \\ Claude S Farah, ${ }^{13}$ Belinda Cochrane, ${ }^{14,15}$ Graham S Hillis (D) , ${ }^{16,17}$ \\ Caroline Polak Scowcroft, ${ }^{18}$ Ashutosh Aggarwal, ${ }^{19}$ Gian Luca Di Tanna, ${ }^{1,2}$ \\ Grace Balicki, ${ }^{1,2}$ Shane Galgey, ${ }^{1,2}$ Christine Jenkins ${ }^{1,2}$
}

To cite: Martin A, Hancox RJ, Chang $\mathrm{CL}$, et al. Preventing adverse cardiac events (PACE) in chronic obstructive pulmonary disease (COPD): study protocol for a doubleblind, placebo controlled, randomised controlled trial of bisoprolol in COPD. BMJ Open 2021;11:e053446. doi:10.1136/ bmjopen-2021-053446

- Prepublication history for this paper is available online. To view these files, please visit the journal online (http://dx.doi. org/10.1136/bmjopen-2021053446).

Received 13 May 2021 Accepted 16 August 2021

D) Check for updates

(c) Author(s) (or their employer(s)) 2021. Re-use permitted under CC BY-NC. No commercial re-use. See rights and permissions. Published by BMJ.

For numbered affiliations see end of article.

Correspondence to Dr Allison Martin; ahumphries@georgeinstitute. org.au

\section{ABSTRACT}

Introduction Heart disease in chronic obstructive pulmonary disease (COPD) is a common but neglected comorbidity. Patients with COPD are frequently excluded from clinical trials of treatments aimed at reducing cardiac morbidity and mortality, which has led to undertreatment of cardiovascular disease in patients with COPD. A particular concern in COPD is the underuse of beta $(\beta)$-blockers. There is observational evidence that cardioselective $\beta$-blockers are safe and may even reduce mortality risk in COPD, although some evidence is conflicting. There is an urgent need to answer the research question: Are cardioselective $\beta$-blockers safe and of benefit in people with moderately severe COPD? The proposed study will investigate whether cardioselective $\beta$-blocker treatment in patients with COPD reduces mortality and cardiac and respiratory morbidity. Methods and analyses This is a double-blind, randomised controlled trial to be conducted in approximately 26 sites in Australia, New Zealand, India, Sri Lanka and other countries as required. Participants with COPD will be randomised to either bisoprolol once daily (range 1.25-5 mg, dependent on tolerated dose) or matched placebo, in addition to receiving usual care for their COPD over the study duration of 24 months.

The study will enrol 1164 participants with moderate to severe COPD, aged 40-85 years. Participants will be symptomatic from their COPD and have a postbronchodilator forced expiratory volume in $1 \mathrm{~s}$ $\left(\mathrm{FEV}_{1}\right) \geq 30 \%$ and $\leq 70 \%$ predicted and a history of at least one exacerbation requiring systemic corticosteroids, antibiotics or both in the prior 24 months.

Ethics and dissemination The study protocol has been approved by the Sydney Local Health District Human Research Ethics Committee at The Concord Repatriation General Hospital.

Trial registration numbers NCT03917914; CTRI/2020/08/027322.

\section{Strengths and limitations of this study}

This will be the first adequately powered randomised controlled trial of a cardioselective $\beta$-blocker, bisoprolol, in addition to usual care in participants with chronic obstructive pulmonary disease (COPD) with all-cause mortality, cardiopulmonary hospitalisations and major adverse cardiac events as a primary, composite endpoint.

- The trial is multicentre and multicountry and participants will have a spectrum of severity of COPD and be included as long as they have had a COPD exacerbation (ECOPD) in the prior 2 years.

- The study has an independent endpoint adjudication committee to determine the underlying cardiac or respiratory cause of death.

- Weaknesses of the study include the impact of the COVID-19 pandemic on both exacerbation rates and COPD hospitalisations, the exclusion of very severe patients with COPD and those who are oxygen dependent.

- The inclusion of hospitalised ECOPD with the other components of the primary outcome may mean that we cannot draw firm conclusions from the results as to whether $\beta$-blockers reduce ECOPD.

\section{INTRODUCTION}

Chronic obstructive pulmonary disease (COPD) is the third-leading global cause of death behind ischaemic heart disease and stroke and the fifth-leading cause of disability affected life years. ${ }^{1}$ In randomised controlled trials (RCTs) of participants with COPD, $30 \%-50 \%$ of deaths are due to cardiac causes, and COPD participants have 
high, often undiagnosed levels of cardiovascular disease (CVD) ${ }^{23} \mathrm{COPD}$ and airflow limitation are risk factors for development of atherosclerosis and CVD, independently of cigarette smoking and air pollution. Even in a population with mild COPD, the Lung Health Study showed that for every $10 \%$ decrease in $\mathrm{FEV}_{1} \%$ predicted, there was a $28 \%$ increase in fatal coronary events and $20 \%$ increase in non-fatal coronary events. ${ }^{4}$ In addition, a recent study demonstrated that COPD exacerbations (ECOPD) more than double the risk of myocardial infarction in the subsequent 5 days. ${ }^{5}$ Subclinical ischaemic events and cardiac arrest occur more frequently after an exacerbation $^{6}$ and cardiac events are strong predictors of mortality in this setting. ${ }^{7}$ In patients hospitalised with an ECOPD in Waikato, New Zealand, ${ }^{8}$ elevated cardiac biomarkers were associated with a 15-times greater likelihood of dying within 30 days of admission than those without raised biomarkers and an $88 \%$ total mortality at 5 years. Despite this, CVD is often undiagnosed and undertreated in patients with advanced COPD.

$\beta$-blockers are indicated for a range of CVDs, but, are frequently withheld from patients with COPD, ${ }^{9}$ due to uncertainty about the potential risk of bronchospasm. To date, most large RCTs of $\beta$-blockers for heart failure and other cardiac diseases have excluded patients with COPD, so their efficacy in COPD is also an area of uncertainty. However, there have been advances in knowledge regarding the distinct airway pathophysiology of COPD, and most observational studies and meta-analyses indicate that patients with COPD do not suffer adverse effects on lung function or symptoms when prescribed cardioselective $\beta$-blockers, ${ }^{10-12}$ even when there is a reversible component to their airflow limitation. Database analyses and retrospective case series suggest that cardioselective $\beta$-blockers are safe ${ }^{10}$ and may be beneficial ${ }^{13-18}$ in patients with COPD. However, the recent $\beta$-Blockers for the Prevention of Acute Exacerbations of COPD (BLOCK COPD) study suggested that the $\beta$-blocker metoprolol may increase the risk of severe and very severe ECOPDs. ${ }^{19}$ Importantly, however, the population in the BLOCK COPD study had a high proportion (40\%) of oxygendependent participants with severe COPD, who may be more sensitive to adverse effects of $\beta$-blockade. ${ }^{20}$ In addition, metoprolol is known to be less cardioselective than some other $\beta$-blockers such as bisoprolol. ${ }^{21-23}$ Faced with this uncertainty about the benefits of $\beta$-blockers in COPD, the fact remains that many people with COPD have cardiovascular indications for the use of $\beta$-blockers, and many patients are currently being treated with them. ${ }^{1624}$ Therefore, it is important that the safety and efficacy of $\beta$-blocker use in people with COPD are further examined.

\section{Rationale}

Since a high proportion of people with COPD die from cardiac events, it is rational to propose that treatments should be trialled that target cardiac comorbidity, whether clinical or subclinical. ${ }^{25}$

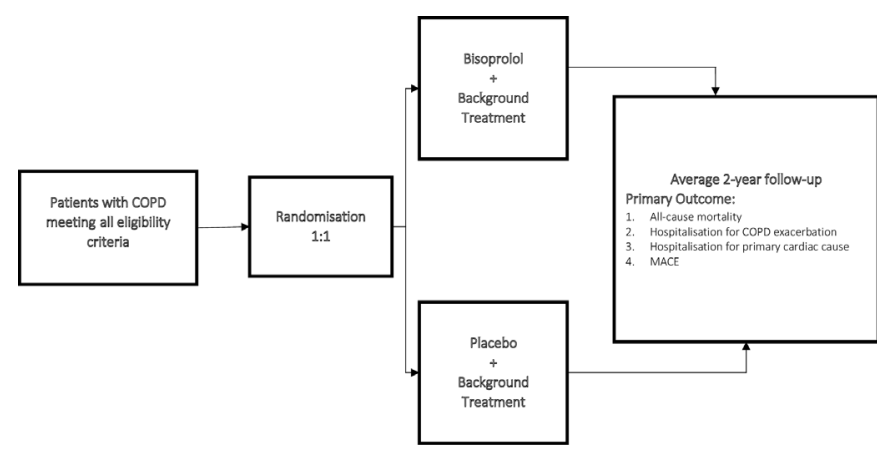

Figure 1 PACE study design. COPD, chronic obstructive pulmonary disease; PACE, preventing adverse cardiac events.

Our group has recently completed a feasibility study assessing the safety and efficacy of commencing the highly cardioselective $\beta_{1}$-blocker, bisoprolol, in people with COPD, and found that it was well tolerated and that a large RCT of cardioselective $\beta$-blockers would be feasible in this participant population. ${ }^{26}$

The hypothesis of the preventing adverse cardiac events in COPD (PACE in COPD) clinical trial is that bisoprolol, taken in addition to usual care in participants with COPD over 24 months, will reduce combined all-cause mortality, cardiac and respiratory hospital admissions and major adverse cardiac events (MACE) compared with matched placebo.

\section{METHODS AND ANALYSIS \\ Trial design and registration}

The PACE in COPD study is a double-blind, multicentre RCT conducted in Australia, New Zealand, India and Sri Lanka with potential for expansion to other countries such as South Africa. Regulatory and ethical approval has not yet been obtained for these additional countries at this point in time. Participants with COPD will be randomised to one of two treatment arms: bisoprolol plus usual COPD care (intervention) or matched placebo plus usual COPD care (control). The design of the study and flow of participants are presented in figure 1

This study will be conducted, analysed and reported according to the Consolidation Standards of Reporting Trials statement for RCTs. ${ }^{27}$ The George Institute for Global Health will act as study sponsor. The trial has been prospectively registered on the ClinicalTrials.gov and the Clinical Trial Registry-India, and other country registries as appropriate.

\section{Participants}

Inclusion and exclusion criteria

Participants will be eligible for this study if they qualify based on all of the criteria detailed in box 1 .

Setting

Participants will be recruited from respiratory and cardiac outpatient clinics, pulmonary rehabilitation services, from 


\section{Box 1 Eligibility criteria}

\section{Inclusion criteria}

- Have provided written informed consent.

- Have chronic obstructive pulmonary disease (COPD) defined by the 2019 Global Initiative for Chronic Obstructive Lung Disease diagnostic criteria.

- Aged $\geq 40$ and $\leq 85$ years.

- Forced expiratory volume in $1 \mathrm{~s}\left(\mathrm{FEV}_{1}\right) \geq 30 \%$ and $\leq 70 \%$ predicted postbronchodilator.

- $\mathrm{FEV}_{1}$ / forced vital capacity $(\mathrm{FVC})<0.7$ postbronchodilator.

- Have had a COPD exacerbation (ECOPD) in the previous 24 months requiring oral corticosteroids, antibiotics or both.

- If taking maintenance oral corticosteroids, dosage is stable and $\leq 10 \mathrm{mg} /$ day for 4 weeks prior to randomisation.

- Resting systolic blood pressure (SBP) $\geq 100 \mathrm{~mm} \mathrm{Hg}$.

- Participants' baseline FEV must not fall $\geq 12 \%$ and $\geq 200 \mathrm{~mL}$ at 2 hours after a test dose of $1.25 \mathrm{mg}$ bisoprolol, and their SBP must be $\geq 100 \mathrm{~mm} \mathrm{Hg}$ at 1 and 2 hours post-test dose.

- For New Zealand participants only, eligible participants must also have a history of CVD, including heart failure, ischaemic heart disease, tachyarrhythmia or hypertension.

\section{Exclusion criteria}

- Concurrent therapy with any other $\beta$-blocker.

- Resting heart rate $<60 \mathrm{bpm}$.

- Unstable left heart failure (ie, symptomatic and/or necessary change in management in the last 12 weeks or in investigator's opinion).

- Clinically significant pulmonary hypertension, which in the investigator's opinion would be a contraindication for $\beta$-blocker therapy.

- Severe end-stage peripheral vascular disease.

- Second or third-degree heart block.

- Currently using or have been prescribed long-term oxygen therapy or resting saturated oxygen level $<90 \%$ when stable.

- Expected survival is less than 12 months, or in the investigator's opinion, the person has such unstable disease (of any type) that maintaining 12 months' participation would be unlikely.

- Clinical instability since a MACE in the previous 12 weeks.

- Lower respiratory tract infection or ECOPD within the last 4 weeks.

- COPD not clinically stable as determined by the investigator.

- In the investigator's opinion, have asthma-COPD overlap or coexistent asthma; or an improvement in $\mathrm{FEV}_{1} \geq 400 \mathrm{~mL}$ postbronchodilator is observed on two occasions.

- Women of childbearing age and capability who are pregnant or breast feeding or those in this group not using adequate birth control.

- Coexistent illness that precludes participation in the study (eg, poorly controlled diabetes, active malignancy).

- Severe end-stage liver disease defined by International Normalised Ratio (INR) $>1.3$ and albumin $<30 \mathrm{~g} / \mathrm{L}$ or portal hypertension/ascites.

- High chance in the opinion of the investigator that the potential participant will not adhere to study requirements.

the inpatient setting and from specialist and primary care referrals.

\section{Procedures}

Potential participants who may be suitable based on the eligibility criteria outlined above will be provided with a current, approved participant information sheet and consent form, by the site personnel and given ample time to review and consider participation before providing written informed consent. Participants will visit the study centres at $0,1,3,6,12,18$ and 24 months. The full schedule of study interventions is outlined in table 1 . Prebronchodilator (baseline only) and postbronchodilator (all visits) spirometry, blood pressure and heart rate (all visits), an ECG (baseline, 1, 3, 12 and 24 months) and 6-minute walk test (baseline and 12 months) will be conducted. Also, the COPD Assessment Test (CAT) ${ }^{28}$ St. George Respiratory Questionnaire (SGRQ) ${ }^{29}$ and EuroQoL Group 5-Dimension self-report questionnaire $\left(\right.$ EQ-5D-5L) ${ }^{30}$ will be completed at baseline $6,12,18$ and 24 months. Participant-reported adverse events will be recorded at all visits. Telephone calls to participants to record adverse events and adherence will be made at 2 weeks, 8 weeks and approximately every 8 weeks in between clinic visits. Adherence to treatment is assessed via tablet counts and self-reported adherence.

\section{Uptitration protocol}

PACE in COPD will use bisoprolol $2.5 \mathrm{mg}$ tablets and matching placebo that will be taken as a half tablet, whole tablet or two tablets according to the uptitration protocol. From baseline to visit 2 (4 weeks), participants will be taking a daily dose of half a tablet of study medication $(1.25 \mathrm{mg}$ bisoprolol or placebo) per day. At the 4-week safety visit, participants will be uptitrated to one whole tablet (2.5 mg bisoprolol or placebo) per day. At visit 3 (12 weeks), the dose of study medication will be increased to two whole tablets ( $5 \mathrm{mg}$ bisoprolol or placebo) per day, provided that participants have not experienced any of the adverse events listed in box 2. If any adverse events occur, the lower dose can be maintained, or the dosage may be reduced. All dose changes are subjected to the investigator's discretion.

\section{Patient and public involvement}

The research question, protocol document and design of the study were informed by feedback obtained from the study's consumer representative, who is a member of the PACE Steering Committee (PSC). Our consumer representative has lived experience from being a carer for a person suffering from COPD and is a COPD patient advocate. Our consumer representative provides ongoing advice on behalf of patients with COPD regarding recruitment and ongoing management of the study. Once the study is complete and analysis has been finalised, a plain language statement will be drafted to explain the outcomes of the study for all randomised participants. This statement will be reviewed by the consumer representative and the central ethics committee prior to dissemination. The burden of the intervention will be assessed by participants via an anonymous qualitative questionnaire when each participant completes the study.

\section{Randomisation, stratification and interventions}

Randomisation will be performed according to a computer-generated algorithm carried out in the IBM 


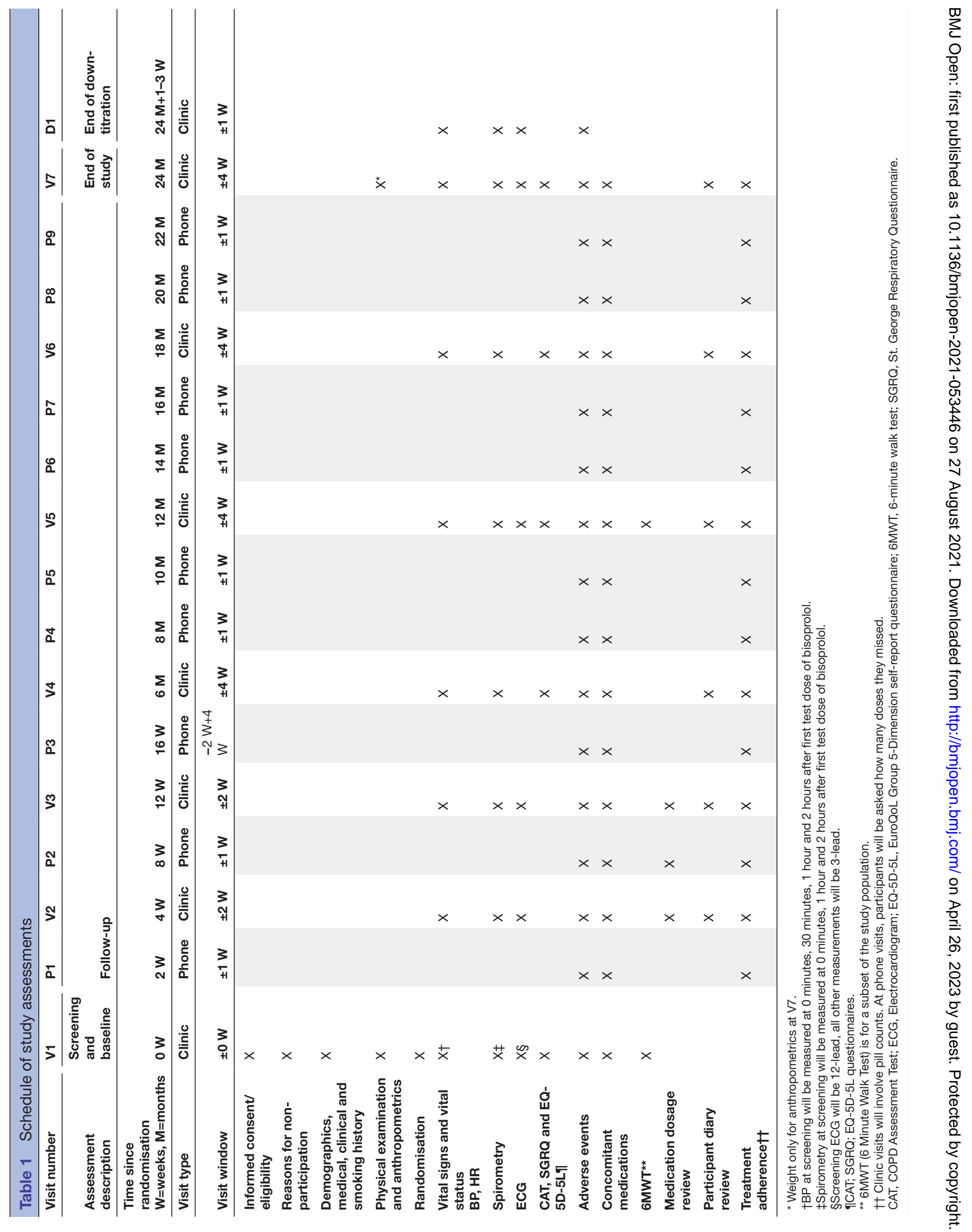


Box 2 Adverse events that preclude uptitration of bisoprolol

- Bronchoconstriction defined as $\geq 12 \%$ and $\geq 200 \mathrm{~mL}$ forced expiratory volume in $1 \mathrm{~s}$ fall from baseline.

- $<100 \mathrm{~mm} \mathrm{Hg}$ or HR $<60 \mathrm{bpm}$.

- Symptomatic postural hypotension.

- Increased wheesing, chest tightness.

- Marked exercise-related fatigue.

Symptoms of peripheral arterial insufficiency.

Clinical Development database. The randomisation list will be concealed and generated centrally using a random combination of four by six permuted blocks within each stratum. Randomisation will be stratified by site, smoking status and previous diagnosis of CVD requiring treatment.

Active and matched placebo will be labelled using blinded kit numbers. Un-blinding will be unlikely, however the Site Principal Investigator has the ability to unblind, after confirmation with the Chief Investigator, within the database. Test dose tablets of bisoprolol, to be given to the participant during screening to test tolerability, will be packaged in a separate container of a half dose $(1.25 \mathrm{mg})$ for each site.

\section{Primary and secondary outcomes}

The primary end point is the time to the first event of a composite outcome, which includes any of: all-cause mortality, hospitalisation for ECOPD or a primary cardiac cause (ischaemia, arrhythmia or heart failure), or a MACE. A MACE event includes cardiovascular death, non-fatal myocardial infarction, or non-fatal stroke. ECOPDs will be graded as moderate (requiring oral corticosteroids, antibiotics or both without hospital admission) or severe (requiring above treatment and hospital admission).

Exploratory outcomes include the annualised ECOPD rate (mild/moderate/severe), time to first moderate/ severe ECOPD, quality of life assessed by the SGRQ, EQ-5D-5L and CAT, clinical spirometry, hospitalisation rate for respiratory and cardiac causes, total number of cardiac events and the number of composite (annualised) cardio-respiratory hospital admissions and MACE.

A within trial cost-effectiveness analysis of the intervention will also be conducted.

\section{Data collection}

All study data will be entered into the electronic case report form (eCRF) hosted by IBM Clinical Development, including clinic and phone visit attendances, study measurements, safety monitoring and all adverse events, as well as the independent adjudication of the primary end points. All data, including source data at sites will be stored for 15 years. Confidentiality will be ensured by only de-identified information being collected and stored centrally. Only authorised sponsor staff will have access to the trial dataset.

\section{Consumer/community involvement}

A consumer representative was involved in the design of this research protocol and has ongoing input into the operational conduct of the study as a member of the Steering Committee. Once the trial is finalised and published, participants will be informed of the results through a plain language statement along with information on the unblinded allocation they received during the trial.

\section{Prohibited concomitant medications}

Participants are not permitted to take other $\beta$-blockers, non-dihydropyridine calcium channel blockers (with the exception of diltiazem, which is at the investigator's discretion), class 1a or 1c anti-arrhythmic agents or topical acetylcholine during the study. If it is deemed clinically necessary to resume a prohibited medication by the treating physician, participants must cease their randomised intervention prior to recommencing the prohibited medication. They will continue with follow-up visits in the study.

\section{Sample size estimation and recruitment target}

The study will recruit 1164 participants to achieve a power of $90 \%$ to detect a risk reduction of $25 \%$ in the intervention vs the control arm (HR=0.75). Assuming a primary end point event rate of 30 per 100 person-years ${ }^{31-33}$ in the control group and that every participant will be followed up for 2 years with up to $10 \%$ lost to follow-up, the study will continue until a total of 514 participants experience a primary endpoint event. The study will be conducted in multiple countries to ensure adequate recruitment is achieved.

\section{Adverse events and safety monitoring}

All adverse events will be recorded at study visits using the eCRF. Adverse events of special interest will include all potential adverse effects of the investigational treatment and COPD-related events, such as light-headedness or falls, increased wheezing, fatigability, abnormal heart rate, worsening respiratory symptoms, respiratory tract infections and including presentation to general practitioners, specialists, or emergency departments for respiratory symptoms. Testing positive for the Severe Acute Respiratory Syndrome Coronavirus-2 will be recorded as an adverse event. If the participant develops coronavirus disease 2019 (COVID-19) and is hospitalised, or if any other serious adverse event (SAE) criteria are met, then this will be classified as an SAE. The standard definition for all other SAEs will be used and data collected and submitted to regulatory agencies as required for each country.

An independent Data Safety and Monitoring Board (DSMB) will monitor the study for safety and efficacy. Five independent, senior clinicians and academics covering respiratory, cardiac and statistical expertise constitute our DSMB membership. The DSMB will perform an ongoing review of predefined safety parameters and overall study 
conduct, with 6-monthly meetings and quarterly SAE line listings. The board will review blinded allocated data (ie, aggregated by treatment group) on participant characteristics and adverse events at regular intervals during the study. The board will be charged with monitoring total mortality, SAEs and making recommendations based on other outcomes such as cause-specific death or non-fatal SAEs. They will principally monitor safety and adverse events without including any formal testing of a treatment effect. Safety analyses presented at DSMB meetings will include summary reports of frequency and differences between treatment groups for deaths, cardiac and respiratory hospitalisations, ECOPDs, MACE and all SAEs.

An independent respiratory or cardiac adjudicator will assess all hospitalisations and deaths reported in the eCRF. If there is disagreement in the causality and/or relatedness of the event between the independent adjudicator and the site investigator, the event will require adjudication by the PACE Adjudication Committee (PAC). The PAC consists of five experienced clinicians who will adjudicate/confirm primary causality of all hospitalisations and deaths. The membership of this committee will be independent of the Chief Investigator team.

Data monitoring will be conducted remotely on a monthly basis and onsite annually at all clinical trial sites by the study sponsor.

\section{Statistical analysis}

The statistical analysis will be by intention-to-treat. The primary analysis will be an analysis of time to any event included in the composite endpoint that occurs first: all-cause mortality, cardiac and respiratory hospital admissions and MACE. The main analysis will consist of a Kaplan-Meier plot and a log-rank test comparing survival times between the $\beta$-blocker and the placebo groups. Sensitivity analyses adjusting for important prognostic factors such as age, sex, smoking status, acute ischaemic event, HEART (history, ECG, age, risk factors and troponin) score (where available), hospitalised ECOPDs or heart failure in the previous 12 months will be conducted using Cox models. In the secondary analyses, all-cause mortality, cardiac or respiratory hospitalisation and MACE will be separately analysed. The rate of ECOPDs will be annualised, analysed by Poisson regression with an overdispersion correction applied if necessary. Continuous variables, such as the CAT scores, SGRQ and $\mathrm{FEV}_{1}$, will be compared by t-tests and mixed models to account for repeated measurements.

\section{Economic evaluation}

We will undertake an Australia-specific trial-based costeffectiveness analysis. Costs will include treatment costs ascertained from participant and study records. Healthcare utilisation will be followed up in both arms of the study based on self-reported general practitioner or hospital attendances and changes in concomitant medications. Health state utilities will be estimated via the EQ-5D-5L and will be used to weight survival up to 24 months to determine quality-adjusted life years. Mean differences in costs and quality-adjusted life years between both treatment groups will be estimated to determine the potential incremental quality-adjusted life year gain from the intervention. Usual deterministic one-way and probabilistic sensitivity analyses will be conducted.

\section{DISCUSSION}

After more than 50 years of widespread use and proven benefit in many contexts, there is limited clinical trial evidence to clarify the effects of cardioselective $\beta$-blockers in people with COPD, with or without clinically evident CVD. To date, there are very few studies targeting cardiovascular risks in participants with COPD, and very few RCTs examining the role of cardioselective $\beta$-blockers to improve the management and outcomes of heart disease in participants with COPD. The 2019 BLOCK COPD ${ }^{19}$ trial investigated the effect of metoprolol compared with placebo on ECOPD, however, the study was stopped early because of futility and concerns of an adverse safety signal. All participants in the BLOCK COPD trial had moderate to severe airflow limitation with $40 \%$ requiring long-term oxygen therapy. Although no statistical association was found for this marker of severity with worse outcomes on metoprolol, these patients were at high risk of severe exacerbation and death. The study reported that metoprolol was associated with an increased risk of severe and very severe exacerbations. However, the design of this trial does not provide any guidance for the use of $\beta$-blockers in COPD populations with less severe disease to prevent cardiac events or ECOPDs. Hence, the target population for the PACE in COPD study are those with an $\mathrm{FEV}_{1} \geq 30 \%$ and $\leq 70 \%$ predicted postbronchodilator and excludes all participants dependent on long-term oxygen therapy, thus enrolling a less severe COPD population, but at similar or greater risk of cardiac events. ${ }^{3435}$ Refer to table 2 for a comparison of the eligibility criteria for the BLOCK COPD and PACE in COPD trials. The addition of lung function and blood pressure criteria after a test dose of bisoprolol to assess eligibility for the PACE trial ensures that participants sensitive to bisoprolol are excluded.

The PACE in COPD trial uses the cardioselective $\beta$-blocker, bisoprolol, with the goal to reduce morbidity and mortality from cardiovascular events and ECOPDs. Differences in cardioselectivity appear to advantage some drugs over others ${ }^{93637}$ in relation to airway outcomes and potentially also to CVD outcomes. Sudden cardiac death is also more common in COPD and may be reduced by $\beta$-blocker therapy. ${ }^{18}$ People with COPD demonstrate evidence of sympathetic overdrive, a consequence of both their disease and acute and chronic $\beta_{2}$-agonist therapy. In heart failure, cardiac $\beta_{1}$-receptors are reduced in number and $\beta_{2}$-receptors are increased, ${ }^{38}$ thus $\beta_{2}$-agonists may further increase adrenergic drive, tachycardia, inotropic contraction and myocardial oxygen consumption, at least in the short term. During an ECOPD, when patients are more hypoxaemic, very large doses of $\beta_{2}$-agonists 
Table 2 Comparison of the BLOCK COPD and PACE in COPD trial eligibility criteria

BLOCK COPD trial ${ }^{18}$ ) PACE in COPD trial

Inclusion

40-85years old

$\mathrm{FEV}_{1}<80 \%$ predicted postbronchodilator

$\mathrm{FEV}_{1} / \mathrm{FVC}$ ratio $<0.7$ postbronchodilator 40-85years old

$\mathrm{FEV}_{1} \geq 30 \%$ and $\leq 70 \%$ predicted postbronchodilator

$\mathrm{FEV}_{1} / \mathrm{FVC}$ ratio $<0.7$ postbronchodilator

Antibiotic/systemic glucocorticoids OR Emergency Department Have had a COPD exacerbation in the previous 24 months (ED)/hospitalisation OR prescription/or use of supplemental requiring oral corticosteroids, antibiotics or both oxygen in previous 12 months

HR 65-120 bpm

$\mathrm{SBP}>100 \mathrm{~mm} \mathrm{Hg}$

$\mathrm{HR} \geq 60 \mathrm{bpm}$

SBP $\geq 100 \mathrm{~mm} \mathrm{Hg}$

Baseline FEV must not fall $\geq 12 \%$ and $\geq 200 \mathrm{~mL}$ at 2 hours after a test dose of $1.25 \mathrm{mg}$ bisoprolol, and participant must have $\mathrm{SBP} \geq 100 \mathrm{~mm} \mathrm{Hg}$ at 1-hour and 2-hour post-test dose.

\section{Exclusion}

Proven indication for use of a $\beta$-blocker, including history of myocardial infarction or revascularisation within 36 months or heart failure with a left ventricular ejection fraction $<40 \%$

Unstable left heart failure, clinically significant pulmonary hypertension, severe end-stage peripheral vascular disease, second or third-degree heart block

Currently using or have been prescribed long-term oxygen therapy, or resting saturated oxygen level $<90 \%$ when stable

BLOCK COPD, Beta-Blockers for the Prevention of Acute Exacerbations of COPD; COPD, chronic obstructive pulmonary disease; FEV forced expiratory volume in $1 \mathrm{~s}$; FVC, forced vital capacity; PACE, preventing adverse cardiac events; SBP, systolic blood pressure.

are often used, and this is associated with persistent biochemical evidence of cardiac dysfunction. ${ }^{39}$ We chose bisoprolol over other cardioselective $\beta$-blockers given its favourable $\beta_{1}$ selectivity and positive findings in database reviews, observational studies and small randomised trials in people with COPD. ${ }^{204041}$

Structural vascular changes have been demonstrated in people with COPD and plaque burden is significantly greater than in matched smokers without COPD or nonsmokers. ${ }^{42}$ Functional vascular changes such as high arterial stiffness have also been found in people with stable COPD and have been shown to increase for up to 8 weeks after exacerbations. Both the presence of plaques and arterial stiffness are established risk factors for cardiovascular events. People with COPD who experience frequent exacerbations have a higher risk of post-ECOPD myocardial infarction and increased arterial stiffness, compared with people with COPD with infrequent exacerbations. This suggests that there may be a cumulative effect of vascular and cardiac damage with frequent exacerbations. Other mechanisms for the association include systemic inflammation, oxidative stress, physiological stresses, adrenergic sensitivity/overdrive during ECOPD and accelerated ageing. ${ }^{42-45}$

There is an urgent need for additional therapies to reduce morbidity and mortality and improve clinical outcomes in people with COPD. Therapies directed at COPD do not improve CVD mortality: despite a marked reduction in ECOPDs, neither long-acting muscarinic antagonists nor combination therapy with inhaled corticosteroids and long-acting $\beta_{2}$ agonists reduce all-cause or
CVD-related mortality. ${ }^{46}$ Moreover, there is an increased risk of fatal and non-fatal cardiovascular events immediately following ECOPDs and so a different strategy is needed, in conjunction with the conventional approach of maximising bronchodilator pharmacotherapy for airway disease. Interventions to reduce cardiac complications during ECOPD along with reducing cardiac risk during stable periods are urgently needed. $\beta$-blocker medications, which have such a major role in improving outcomes in heart failure, need further investigation in the COPD population, a population where their potential benefit is still unrealised, and the extent of cardiac disease remains unrecognised.

There is an urgent need to answer the research question: Are cardioselective $\beta$-blockers safe and of benefit in people with moderately severe COPD? The aim of the PACE in COPD clinical trial is to assess the efficacy and safety of 24 months' therapy with a cardioselective $\beta$-blocker, taken in addition to usual care, in participants with COPD to reduce all-cause mortality, cardiac and respiratory events.

\section{ETHICS AND DISSEMINATION}

The study protocol has been approved by the Sydney Local Health District Human Research Ethics Committee at The Concord Repatriation General Hospital (Protocol Version 7.0, 25 February 2021). In New Zealand, the study protocol has been approved by the Health and Disability Ethics Committee at the Ministry of Health, Wellington. In India, each clinical sites' Institutional Ethics committee 
has individually approved the study protocol. All future amendments will be submitted to the ethics committees and circulated to all clinical sites for governance approval. Clinical trial insurance is provided by the study sponsor as per Medicines' Australia guidelines for compensation.

The trial is registered at ClinicalTrials.gov.

Study findings will be disseminated through presentations at national and international conferences and publications in peer-reviewed journals. Authorship will be determined by the PSC and as per the PACE publication policy.

\section{ROLES AND RESPONSIBILITIES}

Trial sponsor: The George Institute for Global Health, Australia. The Sponsor is responsible for drug procurement, contract development and operational oversight, database development and data collection, ethical conduct and safety reporting.

PSC: Christine Jenkins, Robert Hancox, Catherina Chang, Richard Beasley, Jeremy Wrobel, Vanessa McDonald, Claudia Dobler, Ian Yang, Claude Farah, Belinda Cochrane, Graham Hillis, Caroline Polak Scowcroft, Ashutosh Aggarwal, Allison Martin. The PSC is responsible for the study design, analysis, interpretation of results and writing reports and publications.

DSMB: Professor Eric Bateman (Chair), Professor Anthony Keech (Deputy Chair), A/Professor Katrina Sharples, Professor Gary Anderson, Professor Peter Calverley. The DSMB is an independent, multidisciplinary group including experts in respiratory medicine, cardiology, pharmacology clinical trial methodology and statistics. All members have experience in the conduct and monitoring of RCTs. THE DSMB will meet 6 monthly to ensure ongoing safety surveillance. Two formal interim analyses are planned to assess efficacy and safety, when approximately one-third and two-thirds of the primary endpoint outcomes have been observed. The first of these is expected to occur at approximately 1.5 years after start of the study. The Haybittle-Peto stopping rule based on 3 SDs (interim alpha $=0.27 \%$ ) will be used.

PAC: Professor Lorcan McGarvey (Chair), Professor Claus Vogelmeier, Dr Sundeep Salvi, Professor Michael Williams, Dr Geoffrey Clare. All deaths and cardiac/respiratory hospitalisations will be reviewed by an independent adjudicator. If the independent reviewer agrees with the causality indicated by the principal investigator, no further adjudication is required. If causality is discrepant between the principal investigator and the independent adjudicator, the adverse event is referred to the PAC.

Coordinating centre (Australia/India): Professor Christine Jenkins, Dr Allison Martin, Ms Grace Balicki, Dr Shane Galgey, Mallikarjuna Kunigari. The coordinating centre is responsible for the overall operational conduct of the study in all countries other than New Zealand.

Coordinating Centre (New Zealand): Robert Hancox, Catherina Chang, Christine Tuffery.
Coordinating Centre (Sri Lanka): RemediumOne Pty Ltd

Author affiliations

${ }^{1}$ The George Institute for Global Health, Newtown, New South Wales, Australia

${ }^{2}$ University of New South Wales, Sydney, New South Wales, Australia

${ }^{3}$ University of Otago, Dunedin, New Zealand

${ }^{4}$ Waikato Hospital, Hamilton, New Zealand

${ }^{5}$ Medical Research Institute of New Zealand, Wellington, New Zealand

${ }^{6}$ Fiona Stanley Hospital, Murdoch, Western Australia, Australia

${ }^{7}$ The University of Notre Dame Australia, Fremantle, Western Australia, Australia

${ }^{8}$ University of Newcastle, Callaghan, New South Wales, Australia

${ }^{9}$ John Hunter Hospital, New Lambton Heights, New South Wales, Australia

${ }^{10}$ Liverpool Hospital, Liverpool, New South Wales, Australia

${ }^{11}$ The Prince Charles Hospital, Chermside, Queensland, Australia

${ }^{12}$ The University of Queensland, Saint Lucia, Queensland, Australia

${ }^{13}$ Concord Repatriation General Hospital, Concord, New South Wales, Australia

${ }^{14}$ Campbelltown Hospital, Campbelltown, New South Wales, Australia

${ }^{15}$ Western Sydney University, Penrith, NSW, Australia

${ }^{16}$ Royal Perth Hospital, Perth, Western Australia, Australia

${ }^{17}$ The University of Western Australia, Perth, Western Australia, Australia

${ }^{18}$ Consumer Representative, Canberra, ACT, Australia

${ }^{19}$ Post Graduate Institute of Medical Education and Research (PGIMER), Chandigarh, India

\section{Twitter Vanessa McDonald @nessmcd}

Contributors All authors listed are either members of the PACE Steering Committee or the Coordinating Team and have met regularly to discuss the design of the study and the writing of the study protocol. CJ, RJH, CLC, RB, JW, VM, CCD, IAY, CSF, BC, GH, CPS, AA and AM are members of the PACE Steering Committee and are responsible for the study design, analysis, interpretation of results and writing reports and publications. CJ, AM, GB, SG are all members of the operational study team and are responsible for trial management and planning, safety, monitoring, document creation and implementation and data acquisition. CLC, RB, JW, VM, CCD, IAY, CSF, BC, AA are all site principal investigators and will be responsible for recruitment and retention of trial participants. GLDT is the senior study statistician who assisted with writing the protocol and has oversight over the development of the statistical analysis plan and final analysis of the results. CSP is our consumer representative. GH is responsible for the cardiovascular oversight of the trial design and implementation. All authors have reviewed this protocol manuscript and provided input into its content.

Funding This study is funded by the National Health and Medical Research Council (NHMRC) of Australia, Project Grant APP1163143 and the Health Research Council of New Zealand, Project Grant 19/487.

Competing interests None declared.

Patient and public involvement Patients and/or the public were involved in the design, or conduct, or reporting, or dissemination plans of this research. Refer to the Methods section for further details.

Patient consent for publication Not required.

Provenance and peer review Not commissioned; peer reviewed for ethical and funding approval prior to submission.

Open access This is an open access article distributed in accordance with the Creative Commons Attribution Non Commercial (CC BY-NC 4.0) license, which permits others to distribute, remix, adapt, build upon this work non-commercially, and license their derivative works on different terms, provided the original work is properly cited, appropriate credit is given, any changes made indicated, and the use is non-commercial. See: http://creativecommons.org/licenses/by-nc/4.0/.

\section{ORCID iDs}

Allison Martin http://orcid.org/0000-0001-6065-1188

Richard Beasley http://orcid.org/0000-0003-0337-406X

Claudia C Dobler http://orcid.org/0000-0002-5460-0189

Graham S Hillis http://orcid.org/0000-0003-2417-4673 


\section{REFERENCES}

1 GBD 2015 Chronic Respiratory Disease Collaborators. Global, regional, and national deaths, prevalence, disability-adjusted life years, and years lived with disability for chronic obstructive pulmonary disease and asthma, 1990-2015: a systematic analysis for the global burden of disease study 2015. Lancet Respir Med 2017;5:691-706.

2 Horodinschi R-N, Bratu OG, Dediu GN, et al. Heart failure and chronic obstructive pulmonary disease: a review. Acta Cardiol 2020;75:97-104

3 Almagro P, Boixeda R, Diez-Manglano J, et al. Insights into chronic obstructive pulmonary disease as critical risk factor for cardiovascular disease. Int $J$ Chron Obstruct Pulmon Dis 2020;15:755-64.

4 Anthonisen NR, Connett JE, Enright PL, et al. Hospitalizations and mortality in the lung health study. Am J Respir Crit Care Med 2002;166:333-9.

5 Donaldson GC, Hurst JR, Smith CJ, et al. Increased risk of myocardial infarction and stroke following exacerbation of COPD. Chest 2010;137:1091-7.

6 Odigie-Okon E, Jordan B, Dijeh S, et al. Cardiac injury in patients with COPD presenting with dyspnea: a pilot study. Int J Chron Obstruct Pulmon Dis 2010;5:395-9.

7 Singanayagam A, Schembri S, Chalmers JD. Predictors of mortality in hospitalized adults with acute exacerbation of chronic obstructive pulmonary disease. Ann Am Thorac Soc 2013;10:81-9.

8 Chang CL, Robinson SC, Mills GD, et al. Biochemical markers of cardiac dysfunction predict mortality in acute exacerbations of COPD. Thorax 2011;66:764-8.

9 Neef PA, McDonald CF, Burrell LM, et al. Beta-Blockers are underprescribed in patients with chronic obstructive pulmonary disease and co-morbid cardiac disease. Intern Med J 2016;46:1336-40.

10 Salpeter SR, Ormiston TM, Salpeter EE, et al. Cardioselective beta-blockers for chronic obstructive pulmonary disease. Cochrane Database of Syst Rev 2005;4:CD003566.

$11 \mathrm{Ni}$ Y, Shi G, Wan H. Use of cardioselective $\beta$-blockers in patients with chronic obstructive pulmonary disease: a meta-analysis of randomized, placebo-controlled, blinded trials. J Int Med Res 2012;40:2051-65.

12 Suissa S, Ernst P. $\beta$-blockers for COPD inpatients. Thorax 2012;67:936-7.

13 van Gestel YRBM, Hoeks SE, Sin DD, et al. Impact of cardioselective beta-blockers on mortality in patients with chronic obstructive pulmonary disease and atherosclerosis. Am J Respir Crit Care Med 2008;178:695-700.

14 Rutten $\mathrm{FH}$, Groenwold $\mathrm{RHH}$. $\beta$ blockers for adults with chronic obstructive pulmonary disease. BMJ 2013;347:f7050.

15 Dransfield MT, Rowe SM, Johnson JE, et al. Use of beta blockers and the risk of death in hospitalised patients with acute exacerbations of COPD. Thorax 2008;63:301-5.

16 Quint JK, Herrett E, Bhaskaran K, et al. Effect of blockers on mortality after myocardial infarction in adults with COPD: population based cohort study of UK electronic healthcare records. BMJ 2013;347:f6650.

17 Gottlieb SS, McCarter RJ, Vogel RA. Effect of beta-blockade on mortality among high-risk and low-risk patients after myocardial infarction. N Engl J Med 1998;339:489-97.

18 Narayanan K, Reinier K, Uy-Evanado A, et al. Chronic obstructive pulmonary disease and risk of sudden cardiac death. JACC Clin Electrophysiol 2015;1:381-7.

19 Dransfield MT, Voelker H, Bhatt SP, et al. Metoprolol for the prevention of acute exacerbations of COPD. N Engl J Med 2019;381:2304-14.

20 Ekström MP, Hermansson AB, Ström KE. Effects of cardiovascular drugs on mortality in severe chronic obstructive pulmonary disease. Am J Respir Crit Care Med 2013;187:715-20.

21 Su VY-F, Chang Y-S, Hu Y-W, et al. Carvedilol, bisoprolol, and metoprolol use in patients with coexistent heart failure and chronic obstructive pulmonary disease. Medicine 2016;95:e2427.

22 Mentz RJ, Wojdyla D, Fiuzat M, et al. Association of beta-blocker use and selectivity with outcomes in patients with heart failure and chronic obstructive pulmonary disease (from OPTIMIZE-HF). Am J Cardiol 2013;111:582-7.

23 Salpeter SR, Ormiston TM, Salpeter EE. Cardioselective betablockers in patients with reactive airway disease: a meta-analysis. Ann Intern Med 2002;137:715-25.
24 Stefan MS, Rothberg MB, Priya A, et al. Association between $\beta$-blocker therapy and outcomes in patients hospitalised with acute exacerbations of chronic obstructive lung disease with underlying ischaemic heart disease, heart failure or hypertension. Thorax 2012;67:977-84.

25 Du Q, Sun Y, Ding N, et al. Beta-blockers reduced the risk of mortality and exacerbation in patients with COPD: a meta-analysis of observational studies. PLoS One 2014;9:e113048.

26 Chang CL, Wong C, Beckert L, et al. $\beta$-blockers in exacerbations of COPD: feasibility of a randomised controlled trial. ERJ Open Res 2017;3:00090-2016-2016.

27 Schulz KF, Altman DG, Moher D, et al. Consort 2010 statement: updated guidelines for reporting parallel group randomised trials. BMJ 2010;340:c332.

28 Jones PW, Harding G, Berry P, et al. Development and first validation of the COPD assessment test. Eur Respir J 2009;34:648-54.

29 Jones PW, Quirk FH, Baveystock CM, et al. A self-complete measure of health status for chronic airflow limitation. The St. George's respiratory questionnaire. Am Rev Respir Dis 1992;145:1321-7.

30 Herdman M, Gudex C, Lloyd A, et al. Development and preliminary testing of the new five-level version of EQ-5D (EQ-5D-5L). Qual Life Res 2011;20:1727-36.

31 Shafuddin E, Fairweather SM, Chang CL, et al. Cardiac biomarkers and long-term outcomes of exacerbations of COPD: a long-term follow-up of two cohorts. ERJ Open Res 2021;7:00531-2020-2020.

32 Fermont JM, Fisk M, Bolton CE, et al. Cardiovascular risk prediction using physical performance measures in COPD: results from a multicentre observational study. BMJ Open 2020;10:e038360.

33 Rothnie KJ, Connell O, Müllerová H, et al. Myocardial infarction and ischemic stroke after exacerbations of chronic obstructive pulmonary disease. Ann Am Thorac Soc 2018;15:935-46.

34 Chen W, Thomas J, Sadatsafavi M, et al. Risk of cardiovascular comorbidity in patients with chronic obstructive pulmonary disease: a systematic review and meta-analysis. Lancet Respir Med 2015;3:631-9.

35 Mattila T, Vasankari T, Rissanen $\mathrm{H}$, et al. Airway obstruction and the risk of myocardial infarction and death from coronary heart disease: a national health examination survey with a 33-year follow-up period. Eur J Epidemiol 2018;33:89-98.

36 Hawkins NM, MacDonald MR, Petrie MC, et al. Bisoprolol in patients with heart failure and moderate to severe chronic obstructive pulmonary disease: a randomized controlled trial. Eur $J$ Heart Fail 2009;11:684-90.

37 Jabbour A, Macdonald PS, Keogh AM, et al. Differences between beta-blockers in patients with chronic heart failure and chronic obstructive pulmonary disease: a randomized crossover trial. J Am Coll Cardiol 2010;55:1780-7.

38 Mora MT, Gong JQX, Sobie EA, et al. The role of $\beta$-adrenergic system remodeling in human heart failure: a mechanistic investigation. $J \mathrm{Mol}$ Cell Cardiol 2021;153:14-25.

39 Shafuddin E, Chang CL, Cooray M, et al. Changes in biomarkers of cardiac dysfunction during exacerbations of chronic obstructive pulmonary disease. Respir Med 2018;145:192-9.

40 Lainscak M, Podbregar M, Kovacic D, et al. Differences between bisoprolol and carvedilol in patients with chronic heart failure and chronic obstructive pulmonary disease: a randomized trial. Respir Med 2011;105 Suppl 1:S44-9.

41 Suissa S, Ernst P. Beta-Blockers in COPD: a methodological review of the observational studies. COPD 2018;15:520-5.

42 Stone IS, Barnes NC, Petersen SE. Chronic obstructive pulmonary disease: a modifiable risk factor for cardiovascular disease? Heart 2012;98:1055-62.

43 MacDonald MI, Shafuddin E, King PT, et al. Cardiac dysfunction during exacerbations of chronic obstructive pulmonary disease. Lancet Respir Med 2016;4:138-48.

44 Bernstein D, Fajardo $G$, Zhao $M$. The role of $\beta$-adrenergic receptors in heart failure: differential regulation of cardiotoxicity and cardioprotection. Prog Pediatr Cardiol 2011;31:35-8.

45 Matera MG, Calzetta L, Cazzola M. $\beta$-Adrenoceptor modulation in chronic obstructive pulmonary disease: present and future perspectives. Drugs 2013;73:1653-63.

46 Vestbo J, Anderson JA, Brook RD, et al. Fluticasone furoate and vilanterol and survival in chronic obstructive pulmonary disease with heightened cardiovascular risk (Summit): a double-blind randomised controlled trial. Lancet 2016;387:1817-26. 